\title{
Capsule Commentary on Streed et al., Assessment of Internal Medicine Resident Preparedness to Care for Lesbian, Gay, Bisexual, Transgender, and Queer/Questioning Patients
}

Julia Seay, PhD

University of Miami, Miami, FL, USA.

J Gen Intern Med 34(6):1003

DOI: $10.1007 / \mathrm{s} 11606-019-04907-\mathrm{w}$

(c) Society of General Internal Medicine 2019

$\mathrm{S}$ treed and colleagues examined the effectiveness of an LGBT competency training intervention in modifying knowledge regarding the unique healthcare needs of LGBT populations among a national sample of medical residents. ${ }^{1}$ The web-based cultural competency focused on four areas of LGBT health: (1) sexual orientation and gender identityrelated terminology; (2) health disparities and preventive care issues; (3) unique considerations regarding mental health and substance abuse; and (4) sexually transmitted illnesses. Regardless of year of residency, knowledge increased significantly following the training. ${ }^{1}$

Pre-training knowledge did not differ as a function of residency year, suggesting that, overall, residents may not have been provided with LGBT-focused training as part of their standard training programs. This finding is consistent with prior research showing most medical training programs provide minimal to no LGBT-focused training. ${ }^{2,3}$ In particular, the low pretraining knowledge of LGBT health disparities and preventive care issues among residents is of great concern, given that many LGBT populations experience greater risk for and/or incidence of several preventable diseases, such as HIV, as well as lower use of preventive health services such as cancer screening. ${ }^{4}$ It is encouraging that the completion of the web-based training module led to substantial increases in knowledge in this area, as well as in each of the other three focus areas.

Certainly, increasing knowledge and awareness of care needs unique to LGBT populations is of utmost importance; however, future work must also consider whether such training improves attitudes toward and clinical practices with
LGBT patients, as well as improves health outcomes among LGBT patients. While there are several self-report measures examining physician attitudes and clinical practices toward LGBT patients, researchers' ability to objectively measure changes in clinical practices and potentially ensuing changes in health outcomes is greatly hampered by the lack of sexual orientation and gender identity data collection within medical settings. ${ }^{5}$ Identification of LGBT patients is essential to measuring changes in health outcomes that may result from improved physician training.

Corresponding Author: Julia Seay, PhD; University of Miami, Miami, FL, USA (e-mail: j.seay@med.miami.edu).

\section{Compliance with Ethical Standards:}

Conflict of Interest: The author declare that she does not have a conflict of interest.

Publisher's Note: Springer Nature remains neutral with regard to jurisdictional claims in published maps and institutional affiliations.

\section{REFERENCES}

1. Streed CG, Hedian HF, Bertram A, Sisson SD. Assessment of internal medicine resident preparedness to care for lesbian, gay, bisexual, transgender, and queer/questioning patients. J Gen Intern Med. https://doi. org/10.1007/s12094-016-1587-9.

2. Obedin-Maliver J, Goldsmith ES, Stewart L, et al. Lesbian, gay, bisexual, and transgender-related content in undergraduate medical education. JAMA. 2011;306(9):971-977.

3. McGarry KA, Clarke JG, Landau C, Cyr MG. Caring for vulnerable populations: curricula in U.S. internal medicine residencies. J Homosex. 2008;54(3):225-232.

4. Institute of Medicine. The health of lesbian, gay, bisexual, and transgender people: building a foundation for better understanding. Washington DC: National Academy of Sciences; 2011.

5. Bosse JD, Leblanc RG, Jackman K, Bjarnadottir RI. Benefits of implementing and improving collection of sexual orientation and gender identity data in electronic health records. Comput Inform Nurs. 2018;36(6):267-74. 